\title{
Development and Validation of Reverse Phase-Ultra Performance Liquid Chromatographic Method for Estimation of Related Substances in Febuxostat Drug Substance
}

\author{
Maheswara Reddy Musirike*, Hussain Reddy K and Useni Reddy Mallu \\ Department of Chemistry, Sri Krishnadevaraya University, Ananthapur, Andhra Pradesh- 515003, India
}

\begin{abstract}
A novel high speed, high resolution Reverse phase-UPLC method was developed for the quantification of related substances in Febuxostat drug substance. The separation of drug from the possible impurities was achieved on a Halo $\mathrm{C} 18$ column. The innovative approach of using stationary phase with sub $2 \mu$ particles provides a comprehensive combination of selectivity and speed. $10 \mathrm{mM}$ mono basic potassium phosphate buffer at $\mathrm{pH} 2.7$ and acetonitrile mixture was selected as mobile phase. Flow rate and detection were kept at $0.8 \mathrm{~mL} / \mathrm{min}$ and $320 \mathrm{~nm}$ respectively. The developed UPLC method was subjected to validation parameters. System precision, accuracy, specificity, limit of detection, limit of quantification and linearity were established as per the guidelines recommended by $\mathrm{ICH}$. Stability indicating nature of the method was also performed by exposing the sample under various conditions like acid, base, peroxide and photo stability exposures. Total analysis run time 7.0 minutes indicates the speed and cost saving initiation of the developed method. Using the method one can carry out the quantitative estimation of related substances in Febuxostat drug substance, further the same method can be adopted for determination of drug substance assay also.
\end{abstract}

Keywords: Febuxostat; Ultra performance liquid chromatography; Hyperuricemia with gout; Related compounds; Xanthine oxidase inhibitor

\section{Introduction}

Febuxostat is a xanthine oxidase inhibitor indicated for the chronic management of hyperuricemia in patients with gout [1]. The recommended starting dose of drug is $40 \mathrm{mg}$ once daily. For patients who do not achieve a serum uric acid less than $6 \mathrm{mg}$ per $\mathrm{dL}$ after 2 weeks with $40 \mathrm{mg}$, Uloric $80 \mathrm{mg}$ is recommended. The primary mode of action of a xanthine oxidase inhibitor, achieves its therapeutic effect by decreasing serum uric acid.The objective of this work was to develop a cost effective ultra fast reverse phase UPLC method, the developed method was validated as per regulatory guidelines and transfer the method to quality control lab for analysis of related substances in Febuxostat. As per the literature Febuxostat Determination was done by spectrophotometric method [2] and RP-HPLC [3,4] methods. LC-MS/MS [5-7] assay method was proposed for bioequivalence and pharmacokinetics evaluation. Since this drug is being marketed in domestic and international market the present investigation by the author was to develop a rapid, accurate and precise RP-UPLC method [8-10] for the determination of related substances. The aim of this paper is to develop a cost effective and fast method. The run time for the newly developed method was 7.0 minutes. The innovative approach of using stationary phase with sub $2 \mu$ particles [11-14] provides a comprehensive combination of selectivity and speed. The validation parameters $[15,16]$ provide valuable information on precision accuracy, limit of detection, limit of quantification and linearity of related substances. The method was subjected to validation according to ICH requirements $[17,18]$ (Figure 1).

\section{Materials and Methods}

\section{Instrumentation and reagents}

Test samples and reference standards of Febuxostat were donated by Apotex India pvt ltd. Acetonitrile was purchased from Ultra Scan
Ltd. Ultra performance liquid chromatography from Waters with gradient elution. MilliQ purification system was used to get HPLC grade water. Halo C18, 100 x $2.1 \mathrm{~mm} 2.7 \mu \mathrm{m}$ column purchased from advanced materials technology.

\section{Chromatographic conditions}

The chromatographic separation was achieved on a Halo C18, 100 x $2.1 \mathrm{~mm} 2.7 \mu \mathrm{m}$ Column. Mobile phase consists of $10 \mathrm{mM}$ monobasic phosphate buffer with $0.2 \%$ Triethyl amine and $\mathrm{pH}$ of the solution was adjusted to 2.7. HPLC grade acetonitrile was used as organic modifier. Mobile phase flow rate was kept at $0.8 \mathrm{~mL} / \mathrm{min}$. Gradient program was set as Time/\% of solution B: $0 / 40,3 / 40,3.5 / 70,4 / 75,5 / 80,5.5 / 40$, $7 / 40$. Column temperature was maintained at $50^{\circ} \mathrm{C}$ and detection was carried at $320 \mathrm{~nm}$. Sample compartment was maintained at $10^{\circ} \mathrm{C}$ with an injection volume of $1 \mu \mathrm{L}$.

\section{Preparation of standard and sample}

A mixture of standard solution was prepared by weighing febuxostat and its related compounds to yield a final concentration of $0.10 \%$ of febuxostat and $0.15 \%$ of each Impurity A, Impurity B, Impurity $\mathrm{C}$ and Impurity $\mathrm{D}$ with respect to the sample concentration

*Corresponding author: Maheswara Reddy Musirike, Department of Chemistry, Sri Krishnadevaraya University, Kadiri- Ananthapur Hwy, kandukuru, Anantapur, Andhra Pradesh 515591, India, Tel: 085542 55700; E-mail: musirikemahesh@gmail.com

Received September 16, 2015; Accepted October 16, 2015; Published October 19, 2015

Citation: Musirike MR, Hussain Reddy K, Mallu UR (2015) Development and Validation of Reverse Phase-Ultra Performance Liquid Chromatographic Method for Estimation of Related Substances in Febuxostat Drug Substance. Pharm Anal Acta 6: 431. doi:10.4172/21532435.1000431

Copyright: (c) 2015 Musirike MR, et al. This is an open-access article distributed under the terms of the Creative Commons Attribution License, which permits unrestricted use, distribution, and reproduction in any medium, provided the original author and source are credited. 


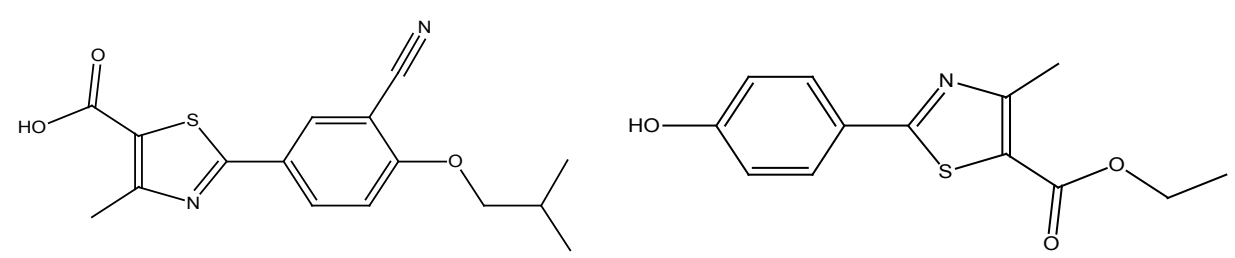

2-(3-cyano-4-isobutoxyphenyl)-4-methylthiazole-5-carboxylic acid Ethyl 2-(4-hydroxy phenyl)-4-methylthiazole-5-carboxylate

(a) Febuxostat (b) Febuxostat Impurity A
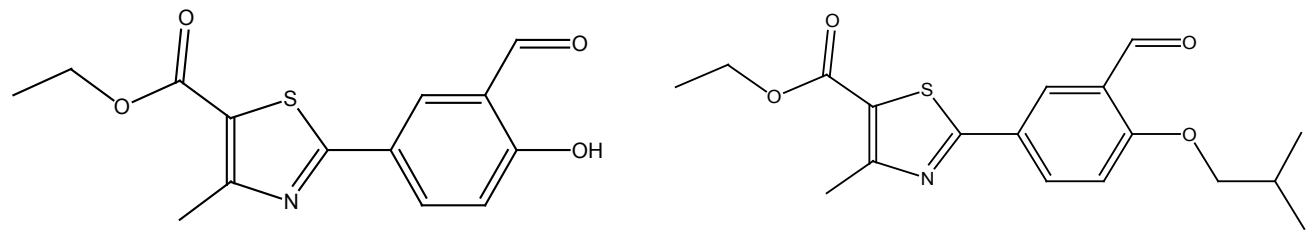

Ethyl 2-(3-formyl-4-hydroxyphenyl)-4-methylthiazole-5-carboxylate Ethyl 2-(3-formyl-4-isobutoxyphenyl)-4-methylthiazole-5-carboxylate

(c) Febuxostat Impurity B (d) Febuxostat Impurity C

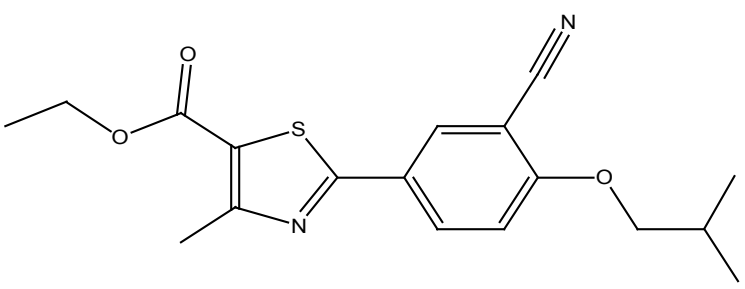

Ethyl 2-(3-cyano-4-isobutoxyphenyl)-4-methylthiazole-5-carboxylate

(e) Febuxostat Impurity D

Figure 1: Chemical structures of febuxostat and its related substances

of $0.5 \mathrm{mg} / \mathrm{ml}$. Buffer and acetonitrile in the ratio 1:4 was used as the diluent for preparation of sample and standard solution.

\section{Method validation}

Specificity: Specificity is the ability of method to measure the analyte response in the presence of its potential impurities. The specificity of the developed RP-UPLC method was demonstrated in presence of sample diluent and its four potential impurities.

System suitability: To ensure system suitability, a standard solution was injected on to the system and verified spectral purity of individual peaks to ensure that no co elution has occurred. Tailing factor $(\mathrm{T})$ column efficiency $(\mathrm{N})$ and resolution $(\mathrm{R})$ were calculated for febuxostat and its related substances.

Precision: Precision of the method was reported by injecting six replicates of standard solution consecutively under the same analytical conditions. The \%RSD of individual peaks was calculated. Intermediate precision of the method was also evaluated using different analyst, different day and different make of instrument in the same laboratory.

Limit of detection (LOD) and limit of quantification (LOQ): The LOD and LOQ for Febuxostat and its related compounds were determined by injecting series of diluted impurity standard solution to a level such that \% RSD was not more than $10 \%$ at LOQ level. Precision carried out at LOQ level by injecting six individual preparations and calculated the \% RSD.

Linearity: Linearity for the related substances method was prepared by serially diluting the impurity stock solution to required concentration levels. The solutions were prepared at five different concentration levels ranging from LOQ to $160 \%$ with respect to specification limits. The calibration curve was drawn by plotting the peak areas of impurities versus its corresponding concentrations. Correlation coefficient of the calibration curve, slope and relative response factors were reported.

Accuracy: Febuxostat sample solution was spiked with impurity standard solutions at three concentration levels corresponding to LOQ, $100 \%$ and $160 \%$ of impurity concentration. The $\%$ recovery was calculated.

\section{Results and Discussion}

\section{Method development and optimization}

Febuxostat and related substances are closely related structures so the main target of the method was to have more specific method to achieve the separation between all impurities from the drug substance effectively and to support routine quality check at competitive time period. The wavelength of detection was selected at $320 \mathrm{~nm}$ as all the related impurities and febuxostat shown maximum absorbance at selected wavelength. Resolution and peak symmetry were good in 
Halo C18. A simple gradient was selected to resolve all the identified impurities in the standard solution and to eliminate interference with diluent and unidentified peaks from sample.

\section{System suitability results}

The peak shape of febuxostat drug substance was found symmetric and well separated by its potential process impurities. A typical system suitability chromatogram of sample diluent, standard solution and test solution chromatograms are shown in Figure 2a-2c.

In the optimized conditions, febuxostat and its related substances were well resolved with a resolution of more than 2.0.The tailing factor is in the range of 1.0-1.2 which indicates symmetry of peaks. Theoretical plates more than 10000 show the efficiency of the column. System suitability parameters for febuxostat and its related substances are tabulated in Table 1.

\section{Method validation results}

Precision: System precision was evaluated by performing six replicate injections for the standard solution at specification level. The $\%$ relative standard deviation of 6 injections was within the acceptable limit.

The obtained results are in the range of 1.4-3.2 which indicates the precision of the system to proceed for analysis. Results are tabulated in Table 2 .

Limit of detection (LOD) and limit of quantification (LOQ): Data obtained for limit of detection (LOD) and limit of quantification (LOQ) of febuxostat drug substance and its related substances are tabulated in Table 3. Sensitivity is the ability of method to detect and quantify the impurities present in the sample accurately. \%RSD for area count of 6 consecutive injections should not be more than $10.0 \%$ at

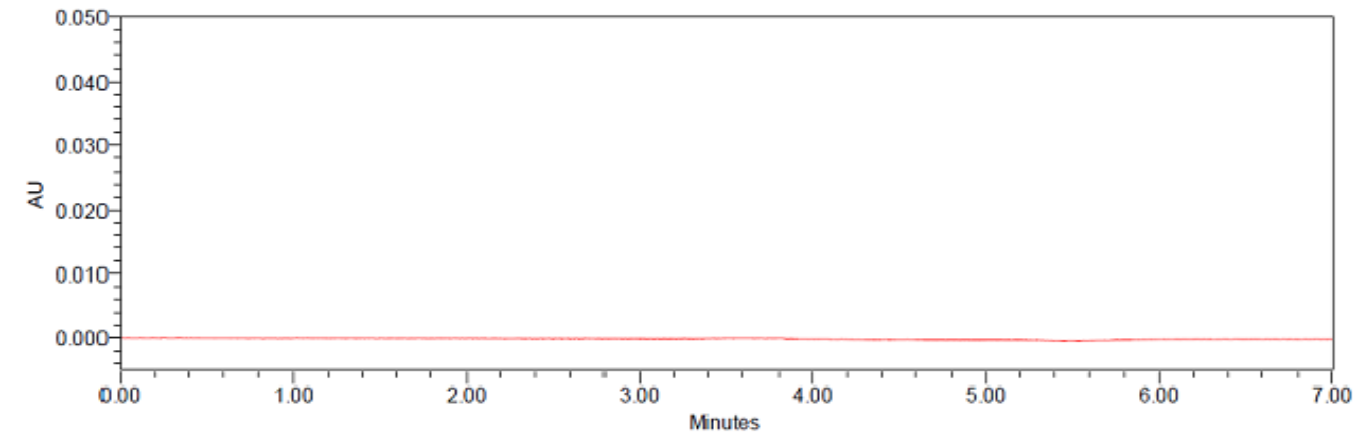

a) Sample diluent

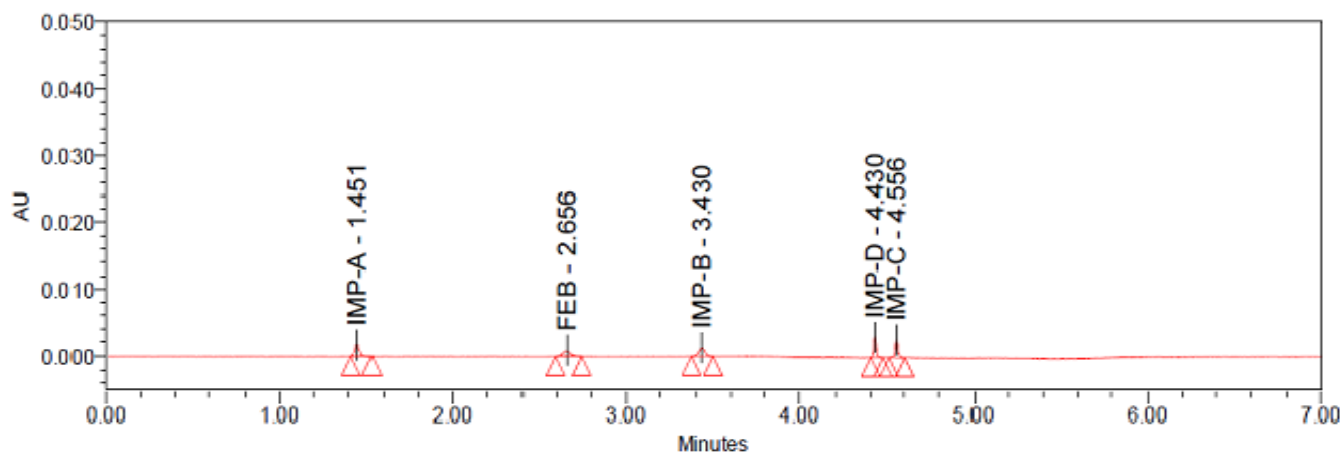

b)Standard solution

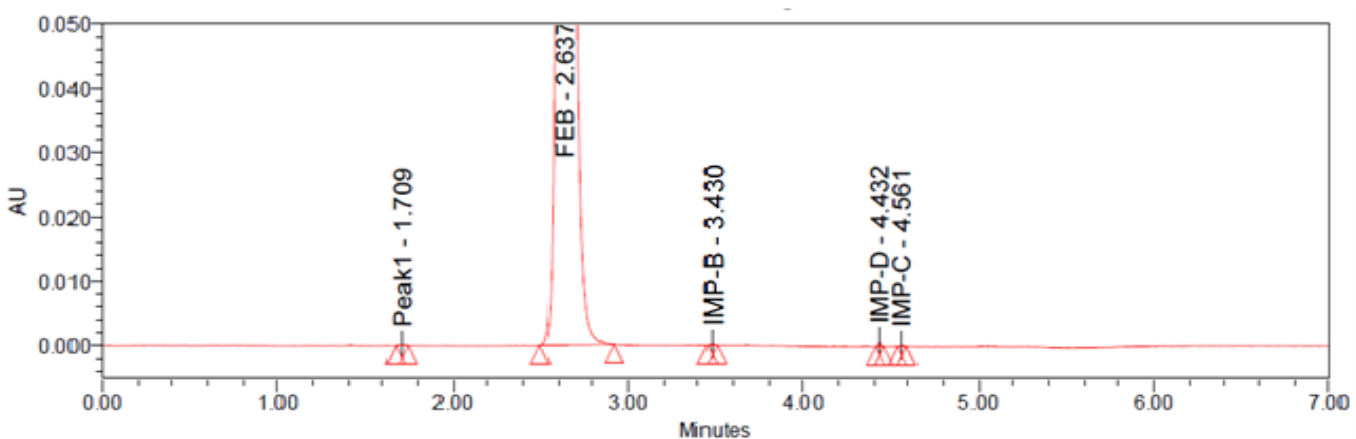

c) Test solution

Figure 2: A typical chromatogram showing the febuxostat (a) sample diluent (b) Standard solution 


\begin{tabular}{|c|c|c|c|c|c|c|}
\hline Component & RT & Resolution & Tailing factor & Plate count & Peak angle & Peak threshold \\
\hline Impurity A & 1.451 & - & 1.2 & 10800 & 3.118 & 5.232 \\
\hline Febuxostat & 2.656 & 15.8 & 1.0 & 13293 & 5.523 & 9.151 \\
\hline Impurity B & 3.430 & 9.2 & 1.0 & 48116 & 4.140 & 6.438 \\
\hline Impurity D & 4.430 & 20.3 & 1.2 & 387969 & 3.243 & 3.632 \\
\hline Impurity C & 4.556 & 4.1 & 1.1 & 341520 & 2.903 & 4.296 \\
\hline
\end{tabular}

Table 1: System suitability results.

\begin{tabular}{|c|c|c|c|c|c|c|}
\hline Inj.\# & Febuxostat & Impurity A & Impurity B & Impurity C & Impurity D & Criteria \\
\hline 1 & 2811 & 3659 & 3386 & 3195 & 3367 & \multirow{9}{*}{ NMT $5 \%$} \\
\hline 2 & 2933 & 3503 & 3431 & 3089 & 3443 & \\
\hline 3 & 2957 & 3538 & 3311 & 3142 & 3591 & \\
\hline 4 & 3075 & 3601 & 3211 & 3091 & 3467 & \\
\hline 5 & 2865 & 3340 & 3449 & 3131 & 3380 & \\
\hline 6 & 2964 & 3463 & 3281 & 3171 & 3348 & \\
\hline Mean & 2934 & 3517 & 3345 & 3136 & 3433 & \\
\hline SD & 90.93 & 111.56 & 92.71 & 42.51 & 90.33 & \\
\hline \%RSD & 3.1 & 3.2 & 2.8 & 1.4 & 2.6 & \\
\hline
\end{tabular}

Table 2: System precision results.

\begin{tabular}{|c|c|c|c|c|}
\hline Inj.\# & Febuxostat & Impurity A & Impurity B & Impurity C \\
\hline LOQ $(\boldsymbol{\mu g} / \mathbf{m l})$ & 0.404 & 0.426 & 0.420 & 0.424 \\
\hline LOD $(\mu \mathbf{g} / \mathbf{m l})$ & 0.13 & 0.142 & 0.140 & 0.428 \\
\hline
\end{tabular}

LOQ-Limit of Quantitation; LOD-Limit of Detection

Table 3: Limit of quantitaion and detection.

\begin{tabular}{|c|c|c|c|c|c|c|c|c|c|c|}
\hline \multirow[b]{2}{*}{ Level } & \multicolumn{2}{|c|}{ Febuxostat } & \multicolumn{2}{|c|}{ Impurity A } & \multicolumn{2}{|c|}{ Impurity B } & \multicolumn{2}{|c|}{ Impurity C } & \multicolumn{2}{|c|}{ Impurity D } \\
\hline & Conc. $(\mu \mathrm{g} / \mathrm{mL})$ & $\begin{array}{l}\text { Area }\left(\mu V^{*}\right. \\
\text { sec })\end{array}$ & Conc. $(\mu \mathrm{g} / \mathrm{mL})$ & $\begin{array}{c}\text { Area }\left(\mu \mathrm{V}^{*}\right. \\
\text { sec })\end{array}$ & Conc. $(\mu \mathrm{g} / \mathrm{mL})$ & $\begin{array}{c}\text { Area }\left(\mu \mathrm{V}^{*}\right. \\
\text { sec })\end{array}$ & Conc. $(\mu \mathrm{g} / \mathrm{mL})$ & $\begin{array}{l}\text { Area }\left(\mu V^{*}\right. \\
\text { sec })\end{array}$ & Conc. $(\mu \mathrm{g} / \mathrm{mL})$ & $\begin{array}{l}\text { Area }\left(\mu \mathrm{V}^{*}\right. \\
\text { sec) }\end{array}$ \\
\hline LOQ & 0.4040 & 1237 & 0.4256 & 1310 & 0.4200 & 1281 & 0.4240 & 1197 & 0.4280 & 1365 \\
\hline $80 \%$ & 0.8080 & 2453 & 0.8512 & 2692 & 0.8400 & 2708 & 0.8480 & 2360 & 0.8560 & 2744 \\
\hline $100 \%$ & 1.0100 & 3143 & 1.0640 & 3712 & 1.0500 & 3532 & 1.0600 & 3185 & 1.0700 & 3383 \\
\hline $120 \%$ & 1.2120 & 3576 & 1.2768 & 4270 & 1.2600 & 4242 & 1.2720 & 3940 & 1.2840 & 4027 \\
\hline $160 \%$ & 1.6160 & 4749 & 1.7024 & 5524 & 1.6800 & 5296 & 1.6960 & 5073 & 1.7120 & 5437 \\
\hline Slope & \multicolumn{2}{|c|}{2886} & \multicolumn{2}{|c|}{3341} & \multicolumn{2}{|c|}{3233} & \multicolumn{2}{|l|}{3115} & \multicolumn{2}{|c|}{3154} \\
\hline $\mathrm{R}^{2}$ & \multicolumn{2}{|c|}{0.9990} & \multicolumn{2}{|c|}{0.9960} & \multicolumn{2}{|c|}{0.9960} & \multicolumn{2}{|c|}{0.9980} & \multicolumn{2}{|c|}{1.0000} \\
\hline RRF & \multicolumn{2}{|c|}{1.00} & \multicolumn{2}{|c|}{0.86} & \multicolumn{2}{|c|}{0.89} & \multicolumn{2}{|c|}{0.93} & \multicolumn{2}{|c|}{0.92} \\
\hline
\end{tabular}

RRF-Relative Response factor, $\mathrm{R}^{2}$ - Correlation Coefficient

Table 4: Linearity results.

LOQ level. The Accuracy at LOQ level should be in the range of 80 to $120 \%$. The Precision at LOQ level and accuracy at LOQ level were found to be within the specified limits.

Linearity: Linearity of the method is to establish a linear relationship of concentration against response. Solutions of febuxostat and its related substances are prepared from LOQ level to $160 \%$ of the specification limit. The correlation coefficient obtained was greater than 0.99 . The regression statistics for febuxostat drug substance and its related substances are tabulated in Table 4.

The result shows that an excellent correlation existed between the peak response and concentration of the analyte and impurities. Relative response factor is established to estimate the factor of impurity response against drug substance response to calculate the content of impurity present in the drug substance.

Accuracy: Accuracy of the method can be determined by spiking known concentrations of standard solution to the sample. The obtained recovery value indicates the trueness of the method to estimate impurities. Related substances of febuxostat spiked to the sample over a concentration range varying from QL to $160 \%$ of their respective target analyte concentrations. The Acceptance criteria for the accuracy are $80 \%$ to $120 \%$.

The obtained percentage recovery value of related substances is in the range of $95.2 \%$ to $102.3 \%$ which declares the method accuracy. Accuracy results are reported in Table 5.

Forced degradation study: Degradation studies were performed to demonstrate stability indicating nature of the method. Febuxostat test sample was exposed to various stress conditions like heat and humidity ( $40^{\circ} \mathrm{C}$ and $70 \% \mathrm{RH}$ for 7 days), thermal $\left(60^{\circ} \mathrm{C}\right.$ for 7 days) and photolytic conditions of fluorescent light $\left(1.2 \times 10^{6} \mathrm{LUX}\right.$ hours $)$, UV light for a total exposure of $200 \mathrm{~W} \cdot \mathrm{hr} / \mathrm{m}^{2}$, acid hydrolysis $\left(0.1 \mathrm{~N} \mathrm{HCl} 80^{\circ} \mathrm{C}\right.$ for $\left.24 \mathrm{Hrs}\right)$, base hydrolysis $\left(0.1 \mathrm{~N} \mathrm{NaOH}, 80^{\circ} \mathrm{C}\right.$ for $\left.24 \mathrm{Hrs}\right)$ and oxidative stress. Testing of peak purity concludes the homogeneity and interference of unidentified impurities with peak of interest. The obtained peak purity value gives a clear indication of separation between stressed impurities with related substances of febuxostat.

Peak obtained in all the stress conditions was homogenous and 


\begin{tabular}{|c|c|c|c|c|c|}
\hline S.NO & Accuracy Level & Impurity A & Impurity B & Impurity C & Impurity D \\
\hline 1 & LOQ & $102.4 \%$ & $87.5 \%$ & $105.2 \%$ & $101.3 \%$ \\
\hline 2 & $100 \%$ & $100.5 \%$ & $85.6 \%$ & $103.1 \%$ & $100.0 \%$ \\
\hline 3 & $160 \%$ & $101.8 \%$ & $95.2 \%$ & $102.3 \%$ & $89 \%$ to $120 \%$ \\
\hline
\end{tabular}

Table 5: Accuracy results.

\begin{tabular}{|c|c|c|c|c|c|c|}
\hline Stress condition & Conc. $\mu \mathrm{g} / \mathrm{mL}$ & Match angle & Match threshold & Purity angle & Purity Threshold & Mass balance \\
\hline Non stressed & 504 & 2.880 & 10.550 & 0.075 & 10.123 & 100 \\
\hline Acid hydrolysis & 520 & 2.542 & 10.235 & 0.012 & 10.225 & 99.8 \\
\hline Base hydrolysis & 510 & 2.325 & 10.835 & 0.085 & 10.522 & 98.9 \\
\hline Oxidation & 530 & 1.987 & 11.123 & 0.023 & 10.995 & 99.9 \\
\hline Heat and humidity & 525 & 1.897 & 11.556 & 0.093 & 10.789 & 99.9 \\
\hline Photo stability & 515 & 2.552 & 11.005 & 0.075 & 10.123 & 99.9 \\
\hline Dry heat & 518 & 2.342 & 11.123 & 0.092 & 10.889 & 99.8 \\
\hline
\end{tabular}

Table 6: Forced degradation studies.

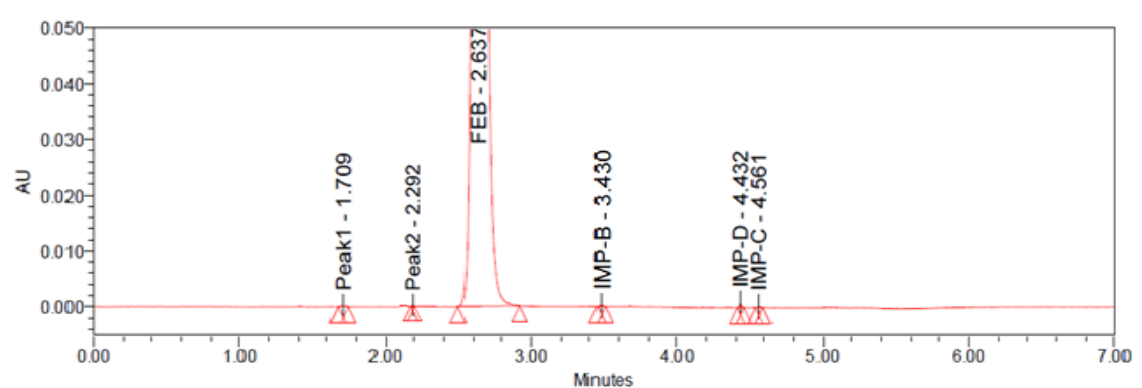

a) Acid Hydrolysis

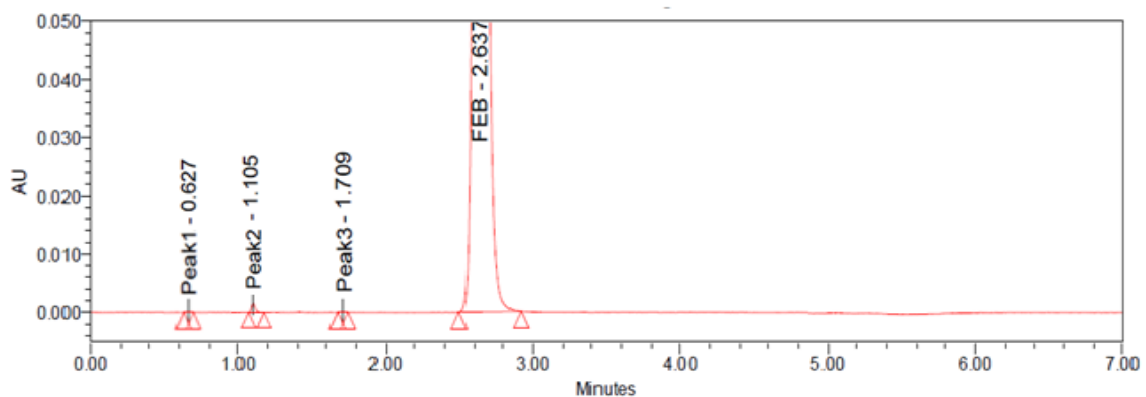

b) Base Hydrolysis

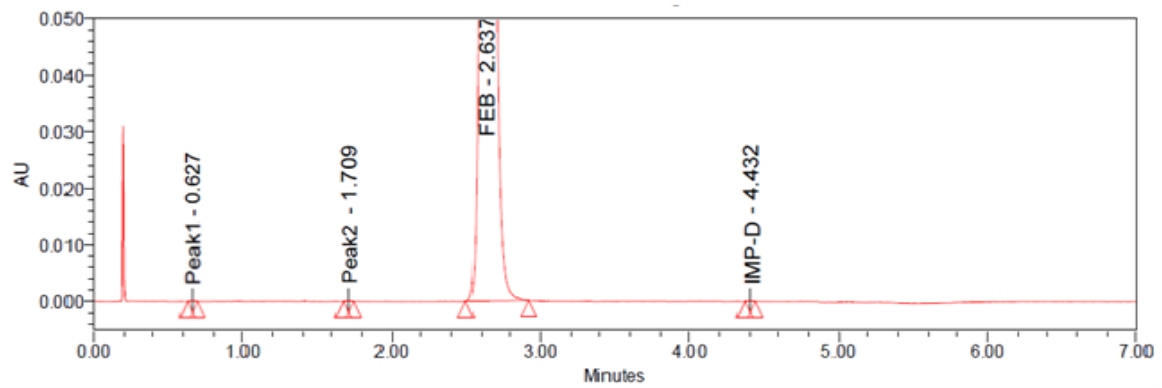

c) Oxidative degradation

Figure 3: A typical chromatogram showing the febuxostat (a) Acid hydrolysis (b) Base Hydrolysis (c) Oxidative degradation.

unaffected by the presence of its degradation impurities, confirming the stability indicating nature of the method. Mass balance also established to match up the sum of impurities with its assay value against reference unstressed sample. The results from forced degradation studies are summarized in Table 6. Figure $3 a-3 c$ indicates stressed sample chromatograms under acidic, basic and peroxide conditions.

\section{Conclusion}

A fast Reverse phase-UPLC method was developed for the 
Citation: Musirike MR, Hussain Reddy K, Mallu UR (2015) Development and Validation of Reverse Phase-Ultra Performance Liquid Chromatographic Method for Estimation of Related Substances in Febuxostat Drug Substance. Pharm Anal Acta 6: 431. doi:10.4172/21532435.1000431

Page 6 of 6

estimation of related substances in Febuxostat drug substance. The developed method was subjected to method validation parameters as recommended by $\mathrm{ICH}$. Stability indicating nature of the method is also established by assessing forced degradation studies. The shorter run time demonstrates that the method is cost effective, time effective and even uses lesser quantity of solvents aiming towards green chemistry. The developed method was specific, precise, accurate and linear to estimate accurate amount of impurities present in the sample. Degradation studies confirmed the homogeneity and free of interferences with the peak of interest.

The method can be adopted to determine the related substances of drug substance in quality control labs. The same procedure can also be used to perform assay of drug substance.

\section{References}

1. Becker MA, Schumacher HR Jr, Wortmann RL, MacDonald PA, Eustace D, et al. (2005) Febuxostat compared with allopurinol in patients with hyperuricemia and gout. N Engl J Med 353: 2450-2461.

2. Paramdeep Bagga, Mohd Salman, Siddiqui $\mathrm{HH}$, Abdul M Ansari, Tariq Mehmood, et al. (2011) A simple UV spectrophotometric method for the determination of Febuxostat in bulk and pharmaceutical Formulations, IJPSR : 2655-2659.

3. Naresh Chandra Reddy M, Chandra Sekhar KB (2012) Estimation of related substances of febuxostat in bulk and 40/80/120mg tablets by RP-HPLC. International Journal of Pharmaceutical, Biological and Chemical Sciences 1: $1-10$.

4. Zhang Cong, Wang Shao-jie, Rong-li MA, Meng Ping, Zhang Tian-hong (2010) Determination of content of febuxostat and its related substances by HPLC, Journal of Shenyang Pharmaceutical University 27: 648-651.

5. Cooper N, Khosravan R, Erdmann C, Fiene J, Lee JW (2006) Quantification of uric acid, xanthine and hypoxanthine in human serum by HPLC for
Pharmacodynamic studies, J Chromatogr B Analyt Technol Biomed Life Sci 837: $1-10$.

6. Dongmei Zhou, David Wilson, Sonny Gunawan, Zancong Shen, Chun Yang Jennifer Yang, et al. A New Method for the Quantitation of Febuxostat in Human Plasma by LC/MS/MS, Abstract session viewer, MP16 Number 384.

7. Khosravan R, Wu JT, Joseph-Ridge N, Vernillet L (2006) Pharmacokinetic Interactions of Concomitant Administration of Febuxostat and NSAIDs. J Clin Pharmacol 46: 855-866.

8. Aimee $\mathrm{N}$ Herrmann, Richard A Henry, importance of controlling mobile phase $\mathrm{pH}$ in reversed phase (RP), Technical bulletin 99-06.

9. Michael E Swartz, Brian J Murphy (2004) Ultra performance liquid chromatography, Tomorrow's HPLC technology today, Lab Plus Internationa 18: 6-9.

10. Lucie Novakova, Ludmila Matysova, Petr Solich (2006) Advantages of application of UPLC in pharmaceutical analysis. Talanta 68: 908-918.

11. David V Mc Calley (2010) Study of the selectivity, retention mechanisms and performance of alternative silica-based stationary phases for separation of ionized solutes in hydrophilic interaction chromatography. Journal of chromatography 1217: 3408-3417.

12. Jerkovich AD, Mellors JS, Jorgenson JW (2003) The use of Micron-Sized Particles in Ultrahigh-Pressure Liquid Chromatography, LCGC 21: 600-610.

13. Wiczling P, Markuszewski MJ, Kaliszan R (2004) Determination of pKa by pH gradient reversed-phase HPLC. Anal Chem 76: 3069-3077.

14. LR Snyder, JJ Kirkland, JL Glajch (1997) Practical HPLC Method Development (2nd edn) 180.

15. (2000) USFDA Documents-Guidance for Industry: Analytical Procedures and Methods Validation.

16. (2005) ICH Q2 (R1): Validation of Analytical Procedures: Text and Methodology.

17. (1996) ICH Q1 B: Stability testing of new drug substances and products.

18. (2006) ICH Q3A (R2): Impurities in new drug substances. 